Check for updates

Cite this: J. Mater. Chem. B, 2021,

9, 1272

Received 27th October 2020,

Accepted 16th December 2020

DOI: 10.1039/d0tb02541k

rsc.li/materials-b

\section{Engineered protein cages for selective heparin encapsulation $\dagger$}

\author{
Salla Välimäki, $\ddagger^{\mathrm{a}}$ Qing Liu, † $^{\mathrm{a}}$ Lise Schoonen, (D) ${ }^{\mathrm{b}}$ Daan F. M. Vervoort, ${ }^{\mathrm{c}}$ Nonappa, (D) d \\ Veikko Linko, (D) ad Roeland J. M. Nolte, (D) ${ }^{b}$ Jan C. M. van Hest (D) ${ }^{b c}$ and \\ Mauri A. Kostiainen (iD *ad
}

\begin{abstract}
A heparin-specific binding peptide was conjugated to a cowpea chlorotic mottle virus (CCMV) capsid protein, which was subsequently allowed to encapsulate heparin and form capsid-like protein cages. The encapsulation is specific and the capsidheparin assemblies display negligible hemolytic activity, indicating proper blood compatibility and promising possibilities for heparin antidote applications.
\end{abstract}

Heparin (Fig. 1a) is a highly anionic polysaccharide belonging to the family of glycosaminoglycans and is widely used as an anticoagulant in surgical practices and thrombotic events. ${ }^{1}$ The anticoagulant activity is based on its ability to bind and subsequently activate antithrombin-III, which leads to the inactivation of vital coagulation cascade substances such as thrombin and factor $\mathrm{Xa}^{2}$ For safe clinical operation, heparin requires an antidote, which can counteract the anticoagulant effect and suppress potential side effects, if needed. ${ }^{3}$ Protamine sulfate is a small arginine-rich cationic protein that binds heparin via electrostatic interactions, and is commonly used for this purpose. ${ }^{2,4}$ This interaction is electrostatic in nature, but nonspecific, and protamine sulfate can cause multiple adverse effects. ${ }^{5}$ This has initiated diverse attempts to develop safer heparin antidotes, such as cationic polymers and small molecules, which all largely rely on the electrostatic binding with heparin. ${ }^{6-12}$

\footnotetext{
${ }^{a}$ Department of Bioproducts and Biosystems, Aalto University, P.O. Box 16100, Aalto FI-00076, Espoo, Finland. E-mail: mauri.kostiainen@aalto.fi

${ }^{b}$ Institute for Molecules and Materials, Radboud University, Heyendaalseweg 135, Nijmegen 6525 AJ, The Netherlands

${ }^{c}$ Department of Bio-Organic Chemistry, Eindhoven University of Technology, Institute of Complex Molecular Systems (ICMS), Het Kranenveld 14, Eindhoven $5600 \mathrm{MB}$, The Netherlands

${ }^{d}$ HYBER Centre, Department of Applied Physics, Aalto University, Aalto FI-00076, Finland

$\dagger$ Electronic supplementary information (ESI) available: Viral capsid protein preparation, purification and characterization methods. Details of TEM, DLS, FPLC, NMR, fluorescence spectroscopy and hemolysis assay. Additional TEM, FPLC and DLS data. See DOI: 10.1039/d0tb02541k

\$ Equal contribution.
}

Although efficient binding can be achieved, cationic platforms often lack specificity to heparin and usually exhibit high cytotoxicity. Furthermore, the complexes formed with heparin are often large and ill-defined aggregates. Therefore, more sophisticated alternatives that would transform heparin into small and welldefined particles, which are biologically inert, are needed. ${ }^{9,12}$

Virus-like particles (VLPs) are protein assemblies derived from wild-type viruses. They lack the viral genome but have similar well-defined capsid structures as native virus particles, and have therefore been explored for biomedical and nanotechnological applications. ${ }^{13-17}$ Indeed, their most appealing features are the accurate sizes and shapes, which are combined with a relatively high stability and versatile modification potential. ${ }^{18}$ The removal of the viral genome also generates a highly confined cavity, which can be harnessed to compartmentalize other nanoscale substances. ${ }^{19-21}$ Cowpea chlorotic mottle virus (CCMV) capsid is one of the most studied VLPs as they can be produced in high yields ${ }^{22}$ and undergo $\mathrm{pH}$ and ionic strength-dependent swelling and assembly, ${ }^{23,24}$ which have therefore been intensively utilized in cargo-loading. ${ }^{25}$

In more detail, CCMV is a plant-infecting icosahedral virus consisting of 180 capsid proteins (CPs) in its native $T=3$ form, leading to a capsid with an outer diameter of $28 \mathrm{~nm}$ and an inner cavity diameter of $18 \mathrm{~nm}$ (Fig. 1b). ${ }^{26}$ Its $T=1$ and pseudo $T=2$ forms consist of 60 and 120 capsid proteins and have diameters of roughly 18 and $22 \mathrm{~nm}$, respectively. ${ }^{27}$ Each CP is formed from 190 amino acids with its N-terminus located on the interior of the capsid. CCMV can be modified in multiple different ways, for example, by functionalizing carboxylic acids or amines on the exterior of the capsid. ${ }^{28,29}$ In addition, the CCMV interior may be altered to enable enhanced cargo loading and encapsulation through electrostatic interactions. ${ }^{30,31}$ To obtain a highly defined modification of the capsid interior, an appealing approach is to engineer the N-termini, which provides site- and number-specificity. For this purpose, an enzymatic method involving a reaction catalyzed by the enzyme Sortase A (SrtA) has been developed. ${ }^{32}$ This benign methodology was first utilized to label the CCMV capsids with a fluorescent tag, but 
a)
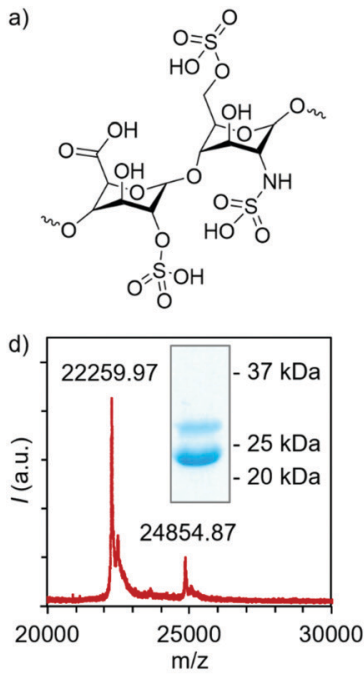

b)
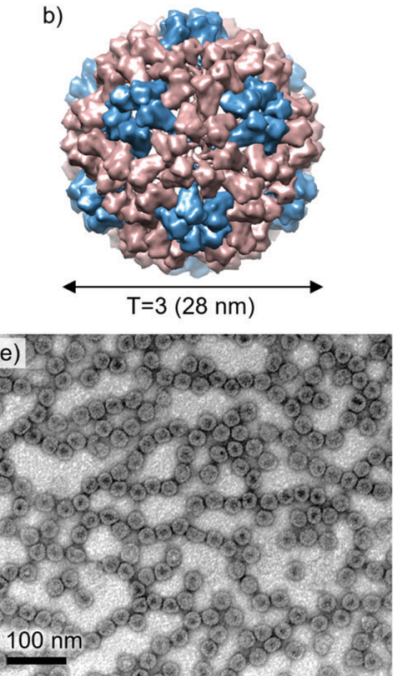

c)
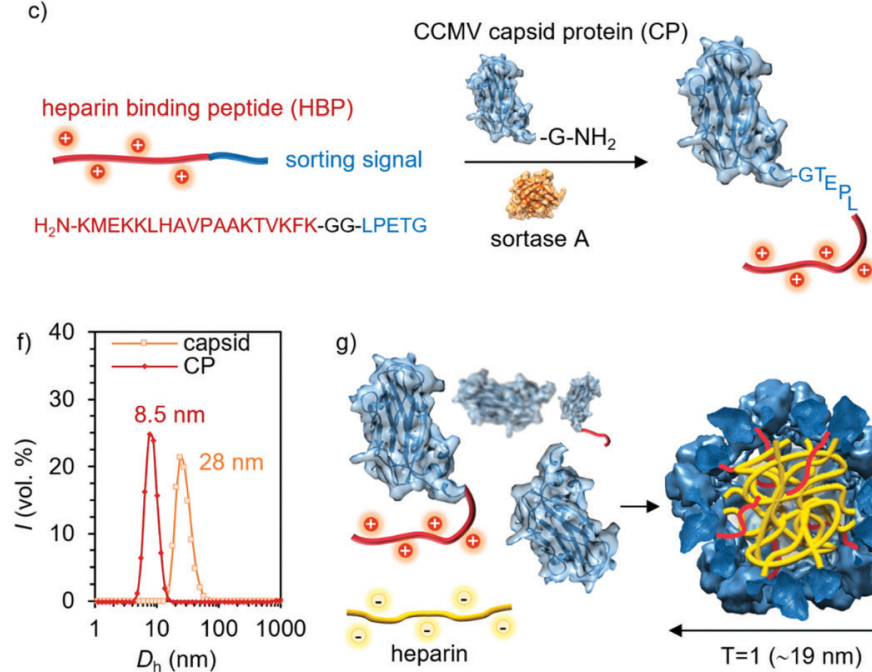

g)

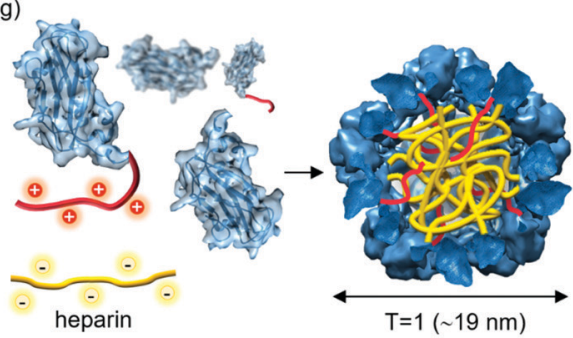

Fig. 1 (a) Structure of the major repeating unit of heparin. (b) Drawing of the CCMV capsid $(T=3)$ structure. (c) Illustration of the Sortase A mediated coupling of HBP to CP. (d) MALDI-TOF spectrum and SDS-PAGE (inset) of HBP modified CPs. (e) TEM image of empty capsids formed by HBP-modified CPs. (f) DLS measurements (volume-weighted size distributions) of the empty capsid and free CP. (g) Schematic representation of heparin (yellow chain) encapsulation by a mixture of CP and HBP modified CP to form $T=1$ structures.

due to the generic nature of the strategy, a wide range of cargos loaded in VLPs can be envisioned. ${ }^{33,34}$

So far, only the exteriors of virions have been employed to display polycationic motifs (poly-Arg) for heparin-binding. ${ }^{35-38}$ However, multiple mutations may yield unstable virions and lead to nonspecific electrostatic interactions with heparin. In this work, we report on the conjugation of a heparin-binding peptide (HBP), i.e. a heparin-specific sequence derived from the fibroblast growth factor having a dissociation constant of $\sim 134 \mathrm{pM}$ with heparin, to the interior of the CCMV capsid. ${ }^{39}$ The binding and packing of heparin into the protein cage was studied with dynamic light scattering (DLS), transmission electron microscopy (TEM), and fast protein liquid chromatography (FPLC). Assemblies were not observed in the presence of two other glycosaminoglycan analogues, demonstrating high selectivity towards heparin. Moreover, no hemolytic activity was detected, implying proper biocompatibility.

The particular HBP was selected based on its high selectivity and affinity to heparin, thus minimizing off-target binding even in complex biological environments. ${ }^{12,39}$ Conjugation of HBP to the CP N-terminus was realized with the SrtA-based method, as illustrated in Fig. 1c. SrtA catalyzes the cleavage of the HBP C-terminal sorting signal (LPETG) after threonine and the subsequent ligation with an N-terminal glycine in the $\mathrm{CP}$. The detailed coupling procedure and characterization of the intermediate conjugates can be found in the ESI $\dagger$ (Fig. S1-S6, ESI $\dagger$ ). The final product was characterized by sodium dodecyl sulfatepolyacrylamide gel electrophoresis (SDS-PAGE) and matrixassisted laser desorption/ionization time of flight (MALDI-TOF) mass spectrometry. Based on the SDS-PAGE and MALDI-TOF analysis, approximately $25 \%$ of the CPs were successfully conjugated with HBP (Fig. 1d and Fig. S7, ESI $\dagger$ ), which corresponds on average to 45 modified CPs per one $T=3$ CCMV capsid. Initially, TEM imaging was employed to investigate the morphology of the assemblies formed by HBP modified CPs alone. Negatively stained spherical VLPs were observed under the TEM microscope, having a diameter of $\sim 28 \mathrm{~nm}$, which corresponds well with the expected dimension of the $T=3$ icosahedral capsids (Fig. 1e and Fig. S9a, ESI $\dagger$ ). The presence of dark regions in the capsid cores indicates that the capsids are empty as the stain is able to enter the voids. ${ }^{40}$ Empty CCMV capsids were then disassembled by dialysis against Tris- $\mathrm{HCl}$ buffer $(50 \mathrm{mM}$, $\mathrm{pH}=7.4$ ). Disassembly was confirmed by TEM and DLS measurements, which showed a size shift from $28 \mathrm{~nm}$ to $8-9 \mathrm{~nm}$ (Fig. 1f and Fig. S9b, ESI $\dagger$ ). The obtained CP solution was then mixed with heparin in different quantities yielding heparinloaded capsids (Fig. 19).

As observed in Fig. 2a-c and Fig. S12, ESI, $\dagger$ the heparinloaded capsid assemblies formed with three different heparin to protein mass ratios $(\rho=0.02,0.22$, and 0.44$)$ all had a roughly spherical morphology and a smaller diameter than the empty $T=3$ capsid $(28 \mathrm{~nm})$. For instance, TEM images indicated an average size of $18.6 \mathrm{~nm}$ at $\rho=0.02$ (Fig. 2d). Similar diameters were observed with the $\rho=0.22(18.6 \mathrm{~nm})$ and $0.44(20.2 \mathrm{~nm})$ samples (Fig. 2d), matching well with the dimension of $T=1$ capsids. The preferential formation of $T=1$ capsids has been observed previously, i.e. when highly negatively charged synthetic polymers $^{40-43}$ and nucleic acids were encapsulated in CCMV VLPs. ${ }^{44,45}$ The particle size has been shown to depend on the molecular weight of the encapsulated compound, and commonly higher molecular weight compounds promote the formation of larger capsids. ${ }^{40,45}$ Unlike empty capsids, the heparin-loaded assemblies did not show dark cores (Fig. 2e), demonstrating that the capsid voids were filled and successfully loaded with heparin molecules. Cryo-TEM images of the heparin-loaded capsids further verified the spherical morphology and $\sim 18 \mathrm{~nm}$ size of the complexes and confirmed that the observed structures are not a result of a drying effect. 

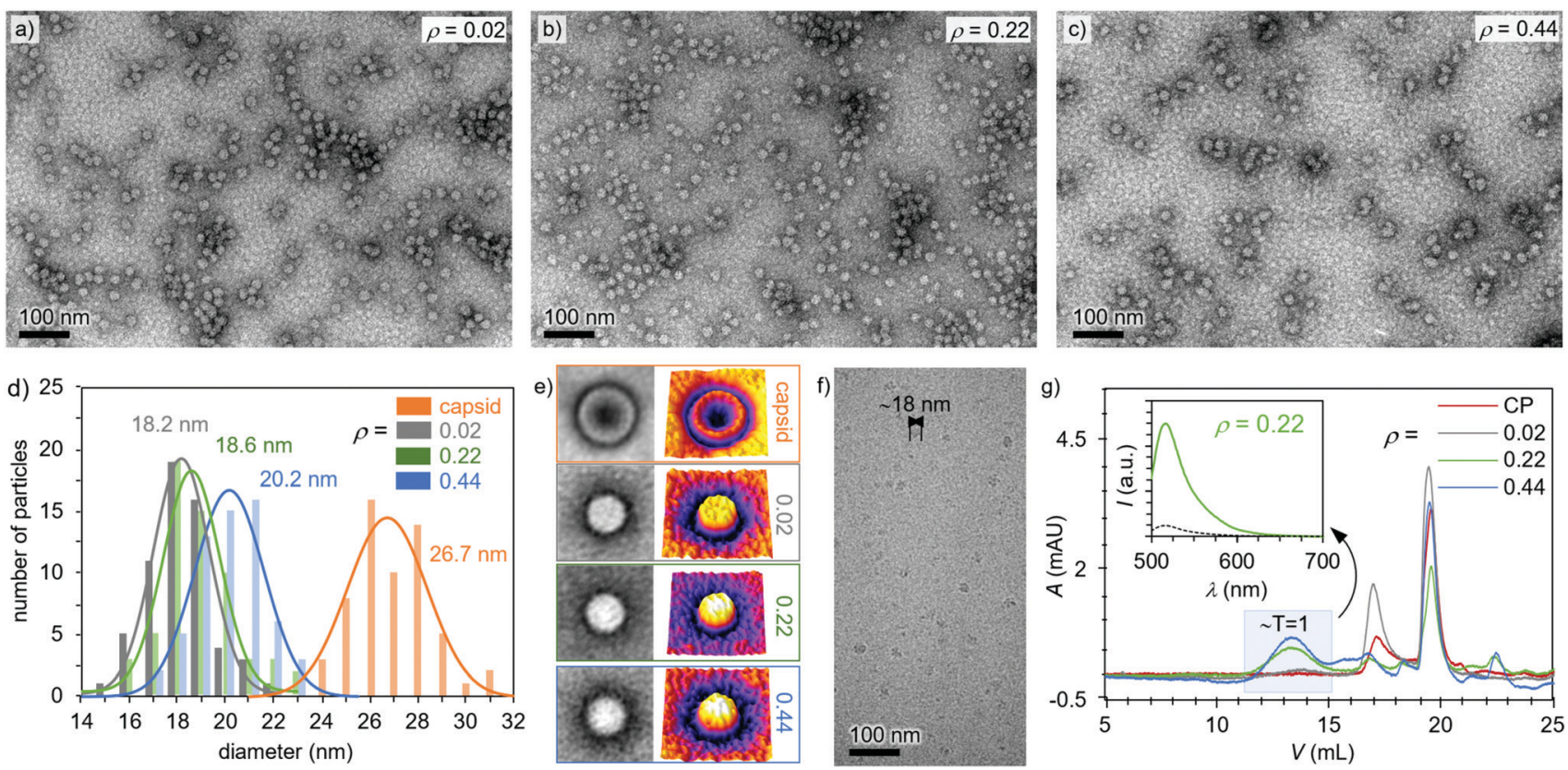

Fig. 2 TEM images of heparin-loaded capsids at (a) $\rho=0.02$, (b) $\rho=0.22$, (c) $\rho=0.44$. (d) Analysis of the dimensions of the complexes $(\rho=0.02-0.44)$ and capsids before disassembly $(\rho=0)$, as derived from the TEM images. Solid curves show Gaussian fits. (e) Averaged $(n=40)$ particle images (size $50 \mathrm{~nm} \times$ $50 \mathrm{~nm}$ ), as taken from panels a-c (left) and their 3D intensity profiles (right). (f) Cryo-TEM image of heparin-loaded capsids at $\rho=0.16$. (g) FPLC chromatogram $(280 \mathrm{~nm}$ ) of free CPs and heparin-loaded capsids $(\rho=0.02-0.44)$. Free CPs elute at $19.6 \mathrm{~mL}$ and $17.1 \mathrm{~mL}$, and $T=1 \mathrm{heparin}$-loaded capsids elute at $13.9 \mathrm{~mL}$. Inset graph shows the fluorescence emission spectrum (excitation at $485 \mathrm{~nm}$ ) of the heparin-loaded capsids ( $\rho=0.22$, green line) and pure heparin-FITC (black dashed line) collected at the $13.9 \mathrm{~mL}$

The effect of the amount of heparin on capsid formation was quantified with fluorescein (FITC) moieties according to a previously published method. ${ }^{46}$ Successful heparin-FITC conjugation was confirmed with FPLC and nuclear magnetic resonance (NMR) spectroscopy (Fig. S8, ESI $\dagger$ ). Fig. $2 g$ shows that the free CPs were eluting at $19.6 \mathrm{~mL}$ and $17.1 \mathrm{~mL}$. Comparison of the peak areas revealed that $71 \%$ of protein was eluting at the higher elution volume (19.6 mL, Fig. S11, ESI $\dagger$ ), which corresponds to the protein dimers. These values are well in line with previously published work conducted under similar conditions where the CP dimers were eluting at $18.5 \mathrm{~mL}^{42}$ In the present studies, another peak at $17.1 \mathrm{~mL}$, corresponding to slightly larger capsomers, was also observed. ${ }^{47}$ The assembled capsids were expected to elute at lower volumes, and for the $\rho=0.02$ sample, a minor broad peak appeared around $13.9 \mathrm{~mL}$ (Fig. $2 \mathrm{~g}$ ), which corresponds to $T=1$ capsids. ${ }^{42}$ When heparin concentration was increased to $\rho=$ 0.22 , the peak fraction at $13.9 \mathrm{~mL}$ increased from $6 \%$ to $37 \%$, showing the formation of more $T=1$ capsids. However, further addition of heparin $(\rho=0.44)$ did not significantly increase the fraction of $T=1$ capsids. Instead it enhanced the amount of the intermediate particles as observed by the larger peak area between $17.1 \mathrm{~mL}$ and $13.9 \mathrm{~mL}$. Additionally, unbound heparin (peak at $22.4 \mathrm{~mL}$ ) started to appear (Fig. S12d, ESI $\dagger$ ), indicating that the excess of heparin did not induce further formation of capsids. This finding is supported by previous studies, which have shown that capsid formation depends on the amount of the encapsulated compound and that high cargo ratios limit capsid formation. ${ }^{41,42}$ Fractions corresponding to the $T=1$ capsids $(13 \mathrm{~mL})$ were collected and investigated with the help of fluorescence spectroscopy measurements. A clear emission spectrum from the fluorescein-conjugated heparin was observed for all the heparin-loaded samples, while that for pure heparinFITC was neglectable, demonstrating that the encapsulations had been successful (Fig. $2 \mathrm{~g}$ inset and Fig. S13, ESI $\dagger$ ).

The selectivity of the heparin encapsulation was evaluated qualitatively with DLS. Studies aimed at encapsulating other negatively charged target molecules were conducted with two glycosaminoglycan analogues, i.e. hyaluronic acid and chondroitin sulfate as well as bovine serum albumin. No size change was observed when these compounds were added to CCMV CPs $(\rho=$ 0.02-0.22), indicating that capsids or other higher-order structures are not formed (Fig. 3a). However, adding heparin in similar mass ratio clearly induced $T=1$ capsid formation, which is visible as a peak between the CP dimers and $T=3$ capsid (Fig. $3 \mathrm{~b}$ and Fig. S14, ESI $\dagger$ ). Adding heparin to the preassembled $T=3$ capsid did not result in any changes in the size distribution indicating that heparin is not bound to the exterior of the capsid. Such observation is expected since the empty capsid remains assembled only at pH 5 in the presence of high electrolyte concentration $(500 \mathrm{mM}$ $\mathrm{NaCl}$ and $10 \mathrm{mM} \mathrm{MgCl}$ ), which efficiently screens electrostatic interactions.

Furthermore, we found negligible hemolytic activity of the CPs up to $0.5 \mathrm{mg} \mathrm{mL}^{-1}$ concentration on red blood cells (RBCs) (Fig. 3c), which is in sharp contrast to the effect of commonly employed protamine sulfate, thus providing a promising strategy in heparin neutralization.

In conclusion, we have shown that CCMV capsid proteins modified with a heparin-binding peptide exhibit specific heparin 

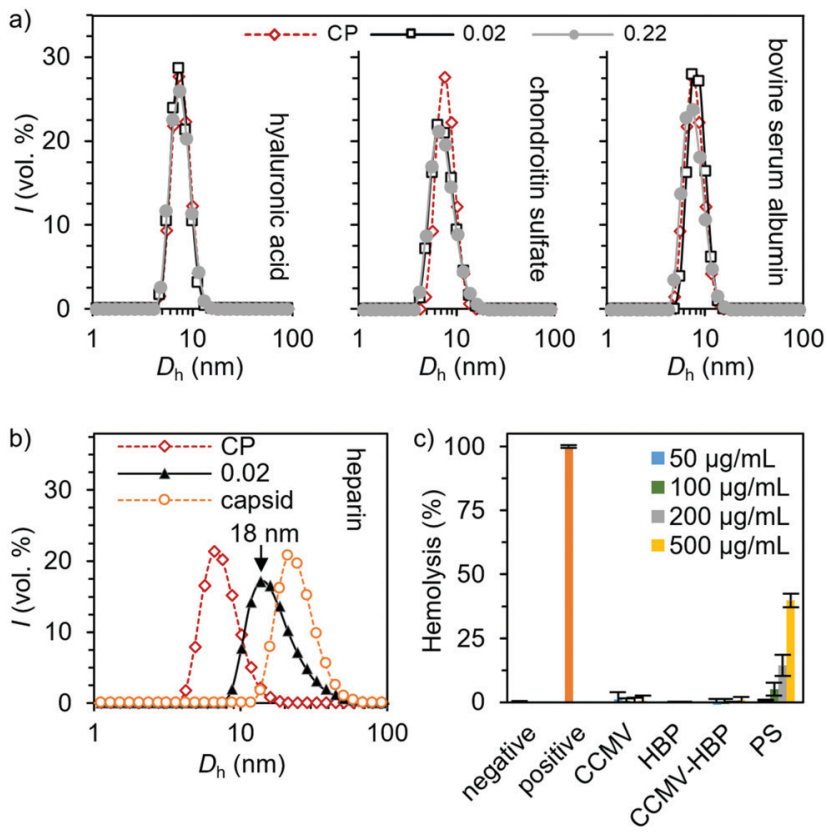

Fig. 3 Selectivity of the capsid assembly induced by (a) hyaluronic acid chondroitin sulfate, bovine serum albumin and (b) heparin, as measured by DLS. (c) Hemolytic activity of the unmodified and HBP-conjugated CPs. Protamine sulfate (PS) was tested for comparison. Solutions of $1 \times$ phosphatebuffered saline (PBS) and $1 \%$ Triton X-100 were used as the negative and positive controls, respectively. Measurements were performed using triplicate samples, and the averaged results with standard deviation are presented.

binding at physiological pH's. Co-assembly with heparin yielded $T=1$ capsids with diameters ranging between 18-20 nm according to the DLS measurements and TEM characterization. FPLC results further confirmed the capsid formation and revealed a heparin concentration-limiting effect on capsid formation. The binding of heparin was proved to be specific as no capsid formation was induced with two other glycosaminoglycan analogs. Moreover, hemolysis assays demonstrated a better biocompatibility than the commercial heparin antidote protamine sulfate. These results indicate that VLPs are promising materials for heparin binding, especially when the binding is further optimized for physiological conditions and after thorough hematologic studies have been conducted.

\section{Conflicts of interest}

The authors declare no conflicts of interest.

\section{Acknowledgements}

We gratefully thank the Academy of Finland (projects 308578, 303804, 267497), the Jane and Aatos Erkko Foundation, the Sigrid Jusélius Foundation and the Emil Aaltonen Foundation for financial support. We acknowledge the provision of facilities at OtaNano - Nanomicroscopy Center (Aalto-NMC) and Bioeconomy Infrastructure. Support was also received from the European Research Council (ERC Advanced Grant 740295 ENCOPOL to R. J. M. N.) and from the Dutch Ministry of Education, Culture and Science (Gravity Program 024.001.035 to R. J. M. N. and J. C. M. v. H.).

\section{References}

1 B. Casu, A. Naggi and G. Torri, Carbohydr. Res., 2015, 403, 60-68.

2 J. Hirsh, T. E. Warkentin, S. G. Shaughnessy, S. S. Anand, J. L. Halperin, R. Raschke, C. Granger, E. M. Ohman and J. E. Dalen, Chest, 2001, 119, 64S-94S.

3 B. Girolami and A. Girolami, Semin. Thromb. Hemostasis, 2006, 32, 803-809.

4 R. Balhorn, Genome Biol., 2007, 8, 227.

5 J. C. Horrow, Anesth. Analg., 1985, 64, 348-361.

6 S. M. Bromfield, E. Wilde and D. K. Smith, Chem. Soc. Rev., 2013, 42, 9184-9195.

7 M. Schuksz, M. M. Fuster, J. R. Brown, B. E. Crawford, D. P. Ditto, R. Lawrence, C. A. Glass, L. Wang, Y. Tor and J. D. Esko, Proc. Natl. Acad. Sci. U. S. A., 2008, 105, 13075-13080.

8 S. Välimäki, A. Khakalo, A. Ora, L.-S. Johansson, O. J. Rojas and M. A. Kostiainen, Biomacromolecules, 2016, 17, 2891-2900.

9 M. T. Kalathottukaren, C. A. Haynes and J. N. Kizhakkedathu, Drug Delivery Transl. Res., 2018, 8, 928-944.

10 V. M. P. Vieira, V. Liljeström, P. Posocco, E. Laurini, S. Pricl, M. A. Kostiainen and D. K. Smith, J. Mater. Chem. B, 2017, 5, 341-347.

11 S. Välimäki, N. K. Beyeh, V. Linko, R. H. A. Ras and M. A. Kostiainen, Nanoscale, 2018, 10, 14022-14030.

12 Q. Liu, S. Välimäki, A. Shaukat, B. Shen, V. Linko and M. A. Kostiainen, ACS Omega, 2019, 4, 21891-21899.

13 Y. Ma, R. J. M. Nolte and J. J. L. M. Cornelissen, Adv. Drug Delivery Rev., 2012, 64, 811-825.

14 P. Singh, M. J. Gonzalez and M. Manchester, Drug Dev. Res., 2006, 67, 23-41.

15 V. Liljeström, A. Ora, J. Hassinen, H. T. Rekola, M. Heilala Nonappa, V. Hynninen, J. J. Joensuu, R. H. A. Ras, P. Törmä, O. Ikkala and M. A. Kostiainen, Nat. Commun., 2017, 8, 671 .

16 M. Rother, M. G. Nussbaumer, K. Rengglic and N. Bruns, Chem. Soc. Rev., 2016, 45, 6213.

17 J. G. Heddle, S. Chakraborti and K. Iwasaki, Curr. Opin. Struct. Biol., 2017, 43, 148.

18 M. Young, D. Willits, M. Uchida and T. Douglas, Annu. Rev. Phytopathol., 2008, 46, 361-384.

19 W. M. Aumiller, M. Uchida and T. Douglas, Chem. Soc. Rev., 2018, 47, 3433-3469.

20 B. Wörsdörfer, K. J. Woycechowsky and D. Hilvert, Science, 2011, 331, 589-592.

21 A. Korpi, E. Anaya-Plaza, S. Välimäki and M. A. Kostiainen, Wiley Interdiscip. Rev.: Nanomed. Nanobiotechnol., 2020, 12, e1578.

22 J.-P. Michel, M. Gingery and L. Lavelle, J. Virol. Methods, 2004, 122, 195-198.

23 L. Lavelle, J.-P. Michel and M. J. Gingery, J. Virol. Methods, 2007, 146, 311-316. 
24 L. Lavelle, M. Gingery, M. Phillips, W. M. Gelbart, C. M. Knobler, R. D. Cadena-Nava, J. R. Vega-Acosta, L. A. PinedoTorres and J. Ruiz-Garcia, J. Phys. Chem. B, 2009, 113, 3813-3819.

25 J. G. Heddle, S. Chakraborti and K. Iwasaki, Curr. Opin. Struct. Biol., 2017, 43, 148-155.

26 J. A. Speir, S. Munshi, G. Wang, T. S. Baker and J. E. Johnson, Structure, 1995, 3, 63-78.

27 J. Tang, J. M. Johnson, K. A. Dryden, M. J. Young, A. Zlotnick and J. E. Johnson, J. Struct. Biol., 2006, 154, 59-67.

28 E. Gillitzer, D. Willits, M. Young and T. Douglas, Chem. Commun., 2002, 2390-2391.

29 E. Gillitzer, P. Suci, M. Young and T. Douglas, Small, 2006, 2, 962-966.

30 I. J. Minten, L. J. A. Hendriks, R. J. M. Nolte and J. J. L. M. Cornelissen, J. Am. Chem. Soc., 2009, 131, 17771-17773.

31 P. A. Suei, Z. Varpness, E. Gillitzer, T. Douglas and M. Young, Langmuir, 2007, 23, 12280-12286.

32 L. Schoonen, J. Pille, A. Borrmann, R. J. M. Nolte and J. C. M. van Hest, Bioconjugate Chem., 2015, 26, 2429-2434.

33 L. Schoonen, S. Maassen, R. J. M. Nolte and J. C. M. van Hest, Biomacromolecules, 2017, 18, 3492-3497.

34 L. Schoonen, R. J. M. Nolte and J. C. M. van Hest, Nanoscale, 2016, 8, 14467-14472.

35 A. K. Udit, C. Everett, A. J. Gale, J. Reiber Kyle, M. Ozkan and M. G. Finn, ChemBioChem, 2009, 10, 503-510.

36 A. J. Gale, D. J. Elias, P. M. Averell, P. S. Teirstein, M. Buck, S. D. Brown, Z. Polonskaya, A. K. Udit and M. G. Finn, Thromb. Res., 2011, 128, e9-e13.
37 H. Y. Cheong, M. Groner, K. Hong, B. Lynch, W. R. Hollingsworth, Z. Polonskaya, J.-K. Rhee, M. M. Baksh, M. G. Finn, A. J. Gale and A. K. Udit, Biomacromolecules, 2017, 18, 4113.

38 J. M. Choi, V. Bourassa, K. Hong, M. Shoga, E. Y. Lim, A. Park, K. Apaydin and A. K. Udit, Mol. Pharmaceutics, 2018, 15, 2997.

39 M. Kan, F. Wang, J. Xu, J. Crabb, J. Hou and W. McKeehan, Science, 1993, 259, 1918-1921.

40 Y. Hu, R. Zandi, A. Anavitarte, C. M. Knobler and W. M. Gelbart, Biophys. J., 2008, 94, 1428-1436.

41 F. D. Sikkema, M. Comellas-Aragonès, R. G. Fokkink, B. J. M. Verduin, J. J. L. M. Cornelissen and R. J. M. Nolte, Org. Biomol. Chem., 2006, 5, 54-57.

42 M. Brasch and J. J. L. M. Cornelissen, Chem. Commun., 2012, 48, 1446-1448.

43 J. B. Bancroft, E. Hiebert and C. E. Bracker, Virology, 1969, 39, 924-930.

44 S. J. Maassen, M. V. de Ruiter, S. Lindhoud and J. J. L. M. Cornelissen, Chem. - Eur. J., 2018, 24, 7456-7463.

45 R. D. Cadena-Nava, M. Comas-Garcia, R. F. Garmann, A. L. N. Rao, C. M. Knobler and W. M. Gelbart, J. Virol., 2012, 86, 3318-3326.

46 Q. Li, L. Ye, A. Zhang and Z. Feng, Carbohydr. Polym., 2019, 211, 370-379.

47 A. Liu, M. Verwegen, M. V. De Ruiter, S. J. Maassen, C. H. Traulsen and J. J. L. M. Cornelissen, J. Phys. Chem. B, 2016, 120, 6352-6357. 